\title{
O papel da atividade física na composição corporal de idosos
}

\section{Marcela Telles Ferreira}

- Nutricionista e professora do Curso de Nutrição do Centro Universitário Municipal de São Caetano do Sul

- Nutricionista do CELAFISCS. Endereço eletrônico: celafiscs@celafiscs.com.br

\section{R E S U M}

Dados têm demonstrado que no Brasil o número de idosos tem crescido exponencialmente. O processo de envelhecimento vem acompanhado de mudanças na composição e no peso corporal. Além disso, a prevalência da obesidade e do sobrepeso também vem crescendo neste segmento da população. Esta tendência produz conseqüências adversas para a saúde, como o aumento do risco para doenças crônicas não transmissíveis. Conseqüentemente, o aumento de tais doenças tem gerado custos elevados para a área da saúde. A atividade física tem sido amplamente empregada, como estratégia para o controle de peso de idosos. Portanto, este trabalho teve como objetivo descrever estudos que relataram o papel da atividade física no controle de peso e na composição corporal de idosos. Os resultados indicaram que para idosos obesos o exercício aeróbico não resulta em perdas consideráveis de peso, devendo estar associado a mudanças nos hábitos alimentares. Já exercício com pesos em doses adequadas produziu mudanças significativas na composição corporal. Evidências epidemiológicas demonstraram que a manutenção de uma atividade física regular contribui para a manutenção ideal do peso, para menores valores de gordura e talvez para a minimização das perdas de massa muscular acompanhadas pelo processo de envelhecimento.

Palavras-chave: atividade física; composição corporal; idosos.

\section{A B S T R A C T}

Several datas have showed that the number of elderly subjects has increased at Brasil. Aging is accompanied by a series of changes in body composition. Besides that, the prevalence of obesity and overweight also has increased in this segment of the population. This tendency produce adverse effects for health including increase risk to develop non-communicable disease. Consequently this increase has produced high costs to the health area. Physical activity has been applied to control body weight among elderly subjects. Thus, these overview summarizes data on the effects of physical activity on body weight and body composition in older adults. It appears that minimal change in weight can be expected in aerobically exercising obese older adults. Several research suggest an inclusion of a diet to an exercise program. On the other hand, numerous studies indicate that resistance training produce considerable changes in body composition. Epidemilogical evidences showed that regular exercise was associated with maintenance ideal weight, body fat less values and perhaps muscle mass minimal losses that accompained aging.

Key words: physical activity; body composition; elderly people. 


\section{INTRODUÇÃO}

No Brasil, atualmente mais de $8 \%$ da população se encontra acima de sessenta anos e este segmento da população tem crescido rapidamente. O envelhecimento vem acompanhado de mudanças no peso e na composição corporal como o aumento gradual do peso durante a meia-idade, seguido por estabilização ou até declínio em idades mais avançadas ${ }^{(30)}$.

Dados sobre a população brasileira de adultos e idosos indicaram que 6,8 milhões de brasileiros são obesos e que 27 milhões apresentam excesso de peso. A prevalência de obesidade e de sobrepeso vem crescendo em idosos e essa tendência produz conseqüências adversas para a saúde. Além disso, há uma associação entre o excesso de peso corporal e o aumento da mortalidade ${ }^{(11)}$.

O papel da atividade física no controle do peso corporal e da massa de gordura tem sido amplamente estudado. Estudos demonstraram que há uma correlação inversa entre a atividade física regular e o IMC. Como a atividade física aumenta o gasto energético e é um fator modificável, seu incremento seria de extrema relevância para se alcançar um determinado peso e composição corporal associados à ótima saúde do idoso ${ }^{(10)}$.

Portanto, a morbi-mortalidade, associada às doenças crônicas não transmissíveis, poderia ser reduzida com a prevenção, incluindo a atividade física como uma mudança no estilo de vida. Descreveremos alguns dos principais estudos acerca do papel da atividade física no controle de peso e na composição corporal em idosos.

\section{O PROCESSO DE ENVELHECIMENTO}

O Brasil vivencia o processo de envelhecimento populacional em decorrência das quedas dos coeficientes de mortalidade e fecundidade. Além disso, esse processo está também associado à melhoria das condições de vida e ao avanço do conhecimento científico, que propicia diagnósticos e tratamentos precoces e colabora para prevenção aos agravos à saúde por meio de vacinas e de medicamentos.

Verificam-se também alterações no perfil da população referente à morbidade e à mortalidade. Enquanto as doenças infecciosas e parasitárias ocupavam lugar de destaque, agora estão sendo substituídas por doenças crônicas não transmissíveis, como hipertensão, diabetes, aterosclerose, obesidade e outras.

De 1995 para 1999, o número de pessoas idosas de 60 anos ou mais de idade cresceu 14,5\%. O lento mas contínuo processo de envelhecimento da população fez com que a participação das pessoas idosas passasse de 7,4\% em 1989 para 8,3\% em 1995 e alcançasse 9,1\% em $1999^{(30)}$.

Já a obesidade teve sua prevalência aumentada na população brasileira, mas também em idosos no período de 1974/75 a 1989, passando, no caso dos idosos, de $2,65 \%$ para $4,98 \%$ em homens e de $9,96 \%$ para $17,89 \%$ em mulheres ${ }^{(11)}$.

Segundo a Organização Mundial da Saúde (OMS), nos países em desenvolvimento, são considerados idosos os indivíduos com 60 anos ou mais.

O envelhecimento pode ser definido como uma série de processos que ocorrem nos organismos vivos e que, com o passar do tempo, leva à perda da adaptabilidade, à alteração funcional e, eventualmente, à morte. A taxa de envelhecimento é a mudança na função dos órgãos e sistemas por unidade de tempo, que apresenta um crescimento exponencial após os 40 anos de idade. Esta taxa é diferente em homens e mulheres. A taxa com que o homem envelhece é baixa com o tempo, enquanto as mulheres envelhecem a uma taxa menor, entre 45 e 60 anos do que entre 70 e 80 anos ${ }^{(18)}$.

\subsection{Obesidade e envelhecimento}

A obesidade é uma condição que aumenta o risco de doenças como hipertensão, diabetes tipo II, dislipidemia, doenças coronarianas, acidente vascular encefálico, osteoartrite, apnéia do sono, problemas respiratórios e câncer do colo, da próstata, de mama e do endométrio. Além disso, o aumento excessivo do peso corporal está associado com o aumento de todas as causas de mortalidade, portanto, o sobrepeso e a obesidade são tratados como uma questão de saúde pública.

Vale ressaltar que, embora sejam necessárias estatísticas mais aperfeiçoadas, é cada vez mais clara a "americanização" dos hábitos alimentares, que, associada à progressiva redução da atividade física diária em razão da mecanização e do avanço tecnológico da sociedade, permite prognosticar gradual aumento de obesos e 
indivíduos com sobrepeso nos diversos segmentos da população brasileira.

Para estudiosos da área, sobrepeso e obesidade são termos distintos, embora relacionados. Sobrepeso é definido como aumento excessivo do peso corporal total, o que pode ocorrer em conseqüência de modificações em apenas um de seus constituintes (gordura, músculo, osso e água) ou em seu conjunto. Mas a obesidade referese especialmente ao aumento na quantidade generalizada ou localizada de gordura em relação ao peso corporal ${ }^{(12)}$.

Segundo BRAY (1997) ${ }^{(4)}$, tanto o sobrepeso quanto a distribuição da gordura corporal são fatores úteis para predizer os riscos à saúde associados à obesidade.

Quando o organismo recebe uma quantidade maior de energia, sob forma de alimento, do que é gasta, o peso corporal aumenta ${ }^{(4)}$. Mas este fator não é a única causa da obesidade. Várias pesquisas apontam que diferenças em fatores específicos podem predispor uma pessoa ao aumento excessivo de peso. Esses fatores incluem: padrões alimentares, meio-ambiente, acondicionamento dos alimentos, imagem corporal, além de diferenças bioquímicas relacionadas à taxa metabólica de repouso, à termogênese de indução dietética, ao nível de atividade espontânea. A temperatura corporal basal, aos níveis celulares de adenosina trifosfato (ATP), lipoproteína lípase e outras enzimas, bem como ao tecido adiposo marrom metabolicamente ativo também contribui para a ocorrência de tal condição.

Afirma, ainda, que se torna difícil separar a causa ou as causas da obesidade em categorias distintas, pois essas provavelmente se superpõem. Parece bastante certo que os procedimentos terapêuticos elaborados até hoje, quer se trate de dieta, cirurgia, medicamentos, métodos psicológicos ou exercício, tanto isoladamente quanto em combinação, não se revelaram particularmente com êxitos na solução.

\subsection{Efeitos do envelhecimento}

Um fenômeno interessante acontece na relação do exercício com o envelhecimento: à medida que o indivíduo envelhece, torna-se menos ativo e suas capacidades físicas diminuem, podendo acarretar em estresse, depressão e, conseqüentemente, o surgimento de doenças crônicas não transmissíveis.

A maioria dos efeitos do envelhecimento ocorre por imobilidade e má adaptação e não por doença crônica. É importante, antes de prescrever atividades físicas para indivíduos da $3^{\mathfrak{a}}$ idade, lembrar os efeitos gerais do envelhecimento que têm sido vastamente descritos pela literatura. Percebe-se que algumas alterações em nível antropométrico relacionadas ao envelhecimento contribuem de forma acentuada no desenvolvimento da obesidade e no padrão de distribuição da gordura corporal.

Tais alterações constituem-se de: incremento no peso, diminuição da massa livre de gordura, diminuição da altura, incremento da gordura corporal, diminuição da massa muscular e diminuição da densidade óssea.

Em nível muscular, as alterações mais freqüentes são: perda de 10 a $20 \%$ na força muscular, diminuição na habilidade para manter força estática, maior índice de fadiga muscular, menor capacidade para hipertrofia, diminuição no tamanho e no número de fibras musculares, diminuição na atividade da ATPase miofibrilar, diminuição das enzimas glicolíticas e oxidativas, diminuição dos estoques de ATP, creatina fosfato, glicogênio, proteína mitocondrial, da velocidade de condução, da capacidade de regeneração e aumento do limiar de excitabilidade da membrana ${ }^{(18)}$.

A redução da massa muscular constitui um fator primário responsável pela perda de força associada à idade e que reflete uma redução na proteína muscular total induzida por inatividade, envelhecimento, ou ambos. A redução no volume muscular pode ser devido ao menor tamanho das fibras, em particular às de contração rápida (tipo II).

Pode haver também uma redução real no número total de fibras musculares. Os estudos eletromiográficos indicam que há perda de neurônios motores funcionantes no idoso. Isso poderia ser responsável por uma perda de fibras musculares, pois a desnervação acarreta atrofia das fibras e eventual substituição por tecido conjuntivo ${ }^{(20)}$.

Esta perda na massa muscular e da força relacionada ao envelhecimento tem sido denominada como sarcopenia ${ }^{(15)}$. 
Em estudo realizado com indivíduos idosos e jovens sedentários, ambos do sexo masculino, o gasto energético nas atividades diárias explicou mais de $75 \%$ da variabilidade da gordura corporal ${ }^{(8)}$.

Perdas na massa muscular também resultam na redução da taxa metabólica basal, da força muscular e do nível de atividade física, que são os fatores causais da diminuição das necessidades energéticas de idosos. Além disso, a diminuição do gasto energético nas atividades diárias também contribui significantemente para a redução das necessidades energéticas. Porém, relatos indicam que as diminuições das necessidades energéticas não são acompanhadas de reduções na ingestão energética, o que resulta em aumento do conteúdo de gordura corporal com o aumento da idade.

Portanto, as alterações na composição corporal, decorrentes do envelhecimento, promovem a diminuição das funções fisiológicas, aumentando a morbi-mortalidade, acarretando, conseqüentemente, custos mais elevados para a área da saúde.

\section{O PAPEL DA ATIVIDADE FÍSICA NO CONTROLE DO PESO EM IDOSOS}

Ao longo dos anos, ocorrem várias mudanças relacionadas ao envelhecimento que influenciam o gasto energético. A atividade física desempenha importante papel no controle do peso corporal em idosos, pois o gasto energético por meio desta pode ser modificado, propiciando o alcance de um peso e composição corporal ideal para ótima saúde e bem-estar.

O gasto energético total é dividido pelo metabolismo basal $(60 \%-70 \%)$, pela termogênese induzida pelo exercício (15\% - 30\%) e pela termogênese induzida pela dieta (10\%). Estima-se que o declínio da taxa metabólica basal seja igual a 1-2\% por década, a partir da $2^{\mathrm{a}}$ à $7^{\mathrm{a}}$ década de vida, e este fato, como já foi relatado, devese, principalmente, à redução da massa magra. A termogênese induzida pela dieta diminui também com o aumento da idade. Outro fator que influencia o gasto energético total é a atividade física voluntária, a qual comumente diminui com o envelhecimento ${ }^{(35)}$.

Além disso, segundo MATSUDO (1992) (19), a atividade física promove efeitos benéficos em indivíduos da 3a idade, tais como: diminuição da gordura corporal, aumento da massa muscular e da força, incremento da densidade óssea, fortalecimento do tecido conetivo, melhora da flexibilidade, além da melhora da auto-estima e da imagem corporal.

TOTH et al. (1999) ${ }^{(35)}$ realizaram revisão acerca do efeito da atividade física na composição corporal de indivíduos idosos. Os autores relataram que as conclusões da maioria dos estudos são transversais, portanto, a verdadeira velocidade das mudanças na composição corporal relacionada com o envelhecimento não está totalmente esclarecida. Além disso, em estudos longitudinais que mediram a atividade física e a composição corporal, ainda permanece incerto qual desses eventos é primário e qual é o secundário.

SERDULA et al. (1999) (31) examinaram a prevalência da tentativa para perder ou manter peso e descreveram as estratégias para controle de peso em adultos americanos. Foram avaliados adultos acima de 18 anos em 49 estados norte-americanos. Os autores verificaram que a atividade física foi o método menos utilizado para perder peso entre os obesos idosos com baixo nível educacional, o que indica a necessidade de melhor comunicação por parte dos profissionais da área da saúde para facilitar a adoção da atividade física para o controle de peso, especialmente neste grupo.

\subsection{Efeitos do exercício aeróbio na composição corporal}

No passado, pensava-se que a obesidade era resultado da ingestão excessiva de alimentos. Portanto, a estratégia de intervenção mais eficaz para o controle de peso consistia em restrição calórica por meio da dieta. Entretanto, evidências atuais indicaram que o aumento excessivo de peso, ao longo da vida, possui uma relação muito mais com a atividade reduzida do que com uma ingestão calórica maior.

O exercício aeróbico regular induz alterações favoráveis no peso e na composição corporal. Observase uma redução da massa de gordura, do peso corporal e da gordura intra-abdominal em idosos que participaram de programas de exercício de endurance.

O fator mais importante em programas de exercício para a redução de peso é o gasto energético total. 
A eficácia de um programa de exercícios para redução ponderal está associada ao grau de obesidade existente no início do programa. Como regra geral, a perda de peso corporal e de massa de gordura é maior em indivíduos que apresentam quantidade de gordura corporal inicial mais alta e que despendem maior quantidade de energia relacionada ao exercício.

Portanto, o programa de exercícios deve estabelecer um equilíbrio calórico negativo para promover uma perda de gordura.

Convém lembrar que o exercício para controle ponderal deve levar em conta alguns fatores como: duração, freqüência e intensidade.

O exercício com intensidade leve à moderada, desde que realizado durante um maior tempo, induz aumento significativo do gasto energético. Trata-se de um aspecto positivo, pois a alta intensidade, na execução dos exercícios, poderá acarretar menor adesão ao programa em virtude principalmente das dores musculares, articulares e fadiga precoce.

O início do programa deve ser lento, progressivo quanto à intensidade e ter regularidade. O exercício mais freqüente é mais eficaz, pois resulta em maior gasto energético.

O exercício aeróbico contínuo, que utiliza grandes grupos musculares e que comporta um gasto energético de moderado a alto, tais como, caminhar, correr, pedalar, pular corda, é ideal para promover a perda de peso. Esse tipo de exercício consome uma quantidade significativa de calorias, estimula o metabolismo lipídico e reduz a gordura corporal. A redução do peso corporal não está estreitamente relacionada com a modalidade do exercício a ser praticado, mas sim a freqüência, a duração e a intensidade com que é executado ${ }^{(20)}$.

BALLOR \& KEESEY (1991) ${ }^{(2)}$ relataram em um estudo de meta-análise, com exercícios acima de 36 semanas de duração, que em homens o exercício aeróbico resultou em perda média de peso corporal de 1,2 kg comparado com o ganho de 1,2 kg de peso nos estudos com levantamento de peso, diminuição média de 1,5 $\mathrm{Kg}(1,7 \%)$ do tecido adiposo, independentemente da modalidade de exercício. Observou-se em mulheres que praticavam a caminhada e o jogging redução do peso corporal de $0,6 \mathrm{~kg}$, comparado com o grupo controle, diminuição de $1,3 \mathrm{Kg}$ da massa de gordura $(1,7 \%)$ e não houve mudança na massa livre de gordura.

Em vários estudos, as diferenças encontradas entre os sexos devem-se ao maior custo energético de atividades equivalentes em homens devido à maior massa corporal.

Em um grupo de 27 senhoras acima de 40 anos, que realizaram, durante o período de 10 semanas, caminhada em esteira, 4 vezes por semana, a 75 a $80 \%$ da freqüência cardíaca máxima, com duração de 40 minutos, observou-se uma diminuição do percentual de gordura, da gordura corporal e aumento da massa livre de gordura ${ }^{(3)}$.

A grandeza com que o exercício aeróbio induz mudanças na massa de gordura está relacionada ao número total de sessões constituintes do programa de exercícios ${ }^{(37)}$.

Em um grupo de dezenove senhoras que participou de um programa de caminhada, 3 vezes por semana, observou-se redução significativa do peso corporal de 0,6 kg em comparação ao grupo controle. No entanto, neste mesmo estudo, os autores promoveram o mesmo programa só que com duração de 5 vezes por semana, para um outro grupo de senhoras $(n=17)$. Ao final do programa o grupo da caminhada 5 vezes por semana, não obteve mudanças significativas no peso corporal. Além disso, nos 2 grupos experimentais houve decréscimo da massa de gordura e do percentual de gordura em relação ao grupo controle. É importante salientar que a duração da caminhada foi de 60 minutos em intensidade equivalente a $60 \% \mathrm{VO}_{2 \text { pico }}$ durante período de 24 semanas ${ }^{(28)}$.

Entretanto, STEFANICK et al. (1998) não verificaram mudanças significativas no IMC e no peso corporal de 47 mulheres pós-menopausa com idade de 50 anos que apresentavam dislipidemia, após um ano de exercício aeróbico. O protocolo de treinamento consistia de sessões de exercício aeróbico supervisionado, 3 vezes por semana durante 1 hora. No $7^{0} \underline{0}$ ou $8^{\circ}$ mês foram adicionadas atividades aeróbicas realizadas em casa como o jogging ou caminhada que equivalessem a pelo menos $16 \mathrm{~km}$ por semana.

Da mesma forma, BUTTERWORTH et al. (1993) ${ }^{(5}$ verificaram que em um grupo de senhoras sedentárias com idade entre 67 e 85 anos que realizaram um programa de caminhada, 5 vezes por semana por 30 - 
40 minutos a $60 \%$ da freqüência cardíaca de reserva durante 12 semanas, não houve mudanças no peso ou na somatória das dobras cutâneas em comparação com um grupo que realizou ginástica calistênica.

Apesar da ausência de evidências que levam a considerar as mudanças na composição corporal em idosos como resultado do declínio do nível de atividade física, os autores concluíram, por meio dos estudos, que o exercício aeróbico reduz o peso corporal, a massa de gordura corporal, pois resulta em um balanço energético negativo e promove pouco efeito na massa livre de gordura ${ }^{(3,28)}$.

Não há evidências, advindas de pesquisas clínicas randomizadas em idosos obesos, de que o exercício aeróbico, como intervenção isolada, pode diminuir significativamente o peso corporal, o percentual de gordura corporal ou a adiposidade central ${ }^{(16)}$. Entretanto, tais exercícios promovem outros benefícios, tais como: melhora da aptidão aeróbica, aumento da sensibilidade à insulina e outros, independentes da perda de peso. Entretanto, parece que há mudanças na composição corporal, por meio de exercícios aeróbicos, em idosos com peso estável ${ }^{(37,34,5)}$.

\subsection{Efeitos do exercício com pesos na composição corporal}

Evidências apontam para uma redução da massa de gordura com o treinamento com peso. Ainda não se sabe quais os mecanismos responsáveis por esta redução. Entretanto, a combinação do aumento das necessidades energéticas para a realização das sessões de treinamento, a elevação da taxa metabólica basal e o possível aumento do "turnover" protéico do músculo esquelético podem, em conjunto, promover o aumento das necessidades energéticas diárias ${ }^{(6,26)}$. É possível que o aumento do número de intervenções que utilizam um programa de treinamento progressivo com pesos se torne uma estratégia útil para a promoção da perda de massa de gordura e para a prevenção do declínio ou até aumento da massa livre de gordura.

Uma vez que o treinamento de resistência pode aumentar ou até preservar o aumento da massa muscular durante a perda de peso, este tipo de exercício promoveria vantagens para idosos que necessitam perder peso.

Embora estudos tenham demonstrado que o treina- mento de endurance é um importante coadjuvante nos programas para perda de peso pelo aumento do gasto energético diário, sua eficácia é relativamente pequena no tratamento para obesidade em idosos. Isso porque muitos idosos sedentários não gastam quantidade significativa de calorias quando realizam o exercício de endurance, em virtude de seu baixo nível de aptidão física. Trinta minutos de exercício aumentam o consumo energético de 100 a 200 calorias, o que representa um valor muito baixo do gasto calórico total ${ }^{(14)}$.

Além disso, o treinamento aeróbico não preserva em grandes proporções a massa magra durante a perda de peso ${ }^{(8)}$.

Exercícios com pesos referem-se a uma modalidade de atividade física sistematizada que pode variar com freqüência, volume (número de exercícios, séries e repetições), tipo de treinamento (isométrico e isocinético), equipamento (máquina de resistência variável ou peso livre) e intensidade. Caracteriza-se como sobrecargas substancialmente altas, utilizadas com o objetivo de melhorar a performance competitiva de atletas numa determinada fase de treinamento, a até sucessivos levantamentos de halteres de $1 / 2 \mathrm{~kg}$ em idosos, exercitando-se sobre uma cadeira e que pretendem melhorar a qualidade de vida ${ }^{(27)}$.

Na reunião anual do "American College of Sports Medicine" foi apresentado o conteúdo do simpósio sobre exercício de resistência para a saúde e a doença. Este simpósio mostrou evidências científicas e a importância do exercício de resistência para a saúde e prevenção de doenças. O exercício de resistência é importante no envelhecimento, na prevenção e na reabilitação de várias doenças crônicas, como a obesidade e o controle de peso, osteoporose, desabilidade e outras.

As diretrizes, segundo essa reunião, incluíram a utilização de exercícios com pesos, 2 a 3 vezes por semana, com 8 a 10 exercícios diferentes, 1 série (moderada) com 10 a 15 repetições para idosos. ${ }^{(25)}$.

Várias organizações conceituadas como "American Heart Association", "American Association of Cardiovascular and Pulmonary Rehabilitation" e "Surgeon General" têm recomendado o treinamento com pesos como parte do programa de atividade física de prevenção e reabilitação ${ }^{(25)}$.

NICHOLS et al. (1993) ${ }^{(23)}$ realizaram um estudo com 
mulheres ativas acima de 60 anos de idade. As senhoras foram divididas em dois grupos: grupo $1(n=18)-$ treinamento de resistência e grupo $2(n=18)$ - grupo controle. O programa de intervenção durou 24 semanas, constituído de 1 série de 8 - 10 repetições (80\% de 1 RM) e freqüência de 3 sessões semanais com intervalo de 48 horas entre cada sessão. À medida que as senhoras conseguiam realizar mais de 10 repetições de um determinado peso, havia um incremento da carga. A composição corporal foi medida por meio de absortometria radiológica de dupla energia, antes e após seis meses. Observou-se que o gasto calórico em exercícios aeróbios, de acordo com um diário de atividade física, não mudou após o programa. O grupo 1 apresentou aumento de 1,5 kg na massa magra, pequena diminuição do percentual de gordura, sem aumento significante do peso corporal. Já o grupo de controle não apresentou alterações. Os autores concluíram que o treinamento de endurance alterou a gordura corporal, porém não modificou a massa magra. Em contraste, nesse estudo, o treinamento com pesos promoveu o aumento na massa magra e diminuição em menor extensão na gordura corporal. Entretanto, a correlação entre as alterações na força e alterações na massa magra foi fraca - não mais que $50 \%$ da variação na força se explica pelas alterações na massa magra, sendo outros fatores responsáveis pelas mudanças observadas na força.

Em recente estudo realizado com 8 mulheres pósmenopausa e 7 homens, com idade entre 61-77 anos, caucasianos, saudáveis e sedentários, com o IMC dentro da normalidade $\left(24,8 \mathrm{~kg} / \mathrm{m}^{2}\right)$, os autores utilizaram um programa de resistência de 26 semanas, utilizando-se de exercícios em aparelhos, 3 vezes por semana, com sessões de 45 minutos, constituídas de 2 séries de 10 repetições, com 2 minutos de repouso entre as séries e intensidade de $65-80 \%$ de 1 RM. Os autores não observaram mudança do peso corporal, porém ocorreu diminuição do percentual de gordura $(3,4 \%)$, redução da massa de gordura $(3,1 \mathrm{~kg})$ e aumento da massa livre de gordura $(2,0 \mathrm{~kg})$. Além disso, verificaram aumento da força $(14,9$ - 49,0 kg), elevação do gasto energético total e do gasto energético de repouso ${ }^{(14)}$. Concluiu-se que, nessa amostra, o treinamento com resistência aumentou o gasto energético total (963 KJ/dia) e permaneceu aumentado após a correção pelo gasto energético durante as sessões de treinamento. O aumento do gasto energético total associou-se com o aumento do gasto energético de repouso e com a atividade física. Este aumento no gasto energético total é relevante para o problema de redução do gasto calórico observado em idosos.

Provavelmente o aumento do gasto energético total após o treinamento com peso deve-se ao aumento da massa livre de gordura e que seria resultante do aumento do gasto energético de repouso, da atividade física e da diminuição do quociente respiratório em resposta ao treinamento ${ }^{(14)}$.

RYAN et al. (1995) ${ }^{(29)}$ avaliaram o efeito de um treinamento de resistência variável constituído de 3 aulas durante 16 semanas a $90 \%$ de 3 RM, sozinho ou acompanhado de perda de peso, com duração de 16 semanas, em 15 mulheres pós-menopausa entre 50 e 69 anos. O grupo foi dividido segundo o IMC: G1 ( $\mathrm{n}=7$; IMC $\left.>27 \mathrm{~kg} / \mathrm{m}^{2}\right)$ e $\mathrm{G} 2\left(\mathrm{n}=8 ; \mathrm{IMC}<27 \mathrm{Kg} / \mathrm{m}^{2}\right)$. O grupo 1 realizou o programa de treinamento de resistência mais perda de peso, enquanto o grupo 2, apenas treinamento de resistência. Os autores não verificaram mudança no peso corporal e na massa de gordura do grupo 2, entretanto, no grupo 1, houve redução do peso, da massa gorda e do percentual de gordura. Verificaram também aumento de massa livre de gordura e da força muscular em ambos os grupos. Quando os grupos foram agregados, observou-se aumento da taxa metabólica basal.

Estudos atuais realizados por RASO (2000) (27) verificaram o efeito de um programa sistematizado de exercícios com pesos na adiposidade, no IMC e no peso corporal de mulheres com média de idade de 65,8 anos. O programa era composto de 3 séries de 10 repetições a 50\% RM para 6 exercícios, com freqüência de 3 vezes por semana durante 12 semanas. Quando comparou o grupo com peso com um grupo que fazia atividade aeróbica, o autor observou que houve uma diminuição 
do peso $(1,5 \%)$ e do IMC $(1,6 \%)$ no grupo com peso e de 3,3\% e 2,8\% no grupo aeróbico, respectivamente. Quanto à adiposidade, houve uma variação em termos percentuais similar entre ambos os grupos $(-9,2 \%$ grupo com peso e -10,5\% grupo aeróbico).

No entanto, outros estudos não encontraram mudanças no peso corporal e taxa metabólica basal após o treinamento com pesos ${ }^{(7,35)}$.

Portanto, o treinamento com pesos promoveu queda da massa de gordura e aumento da massa livre de gordura e tendência de aumento da taxa metabólica basal independente do aumento da idade ${ }^{(6,23,26,14,29)}$.

Parece que o protocolo combinado de treinamento de endurance mais treino com resistência preserva a massa magra e aumenta a taxa metabólica basal mesmo com perda de peso em mulheres com sobrepeso.

As modificações na composição corporal e na taxa metabólica basal observadas com o treinamento de resistência são de grande importância para o controle de peso a longo prazo.

Embora não haja estudos com bases epidemiológicas que sugerem que o treinamento de resistência diminuiria a obesidade ou a morbi-mortalidade relacionada à obesidade, principalmente no público feminino, devido ao fato de não ser uma atividade praticada usualmente pela população, a maioria dos estudos demonstrou que o treinamento com peso em idosos saudáveis promove aumento do gasto energético e da velocidade de oxidação de lipídios, mesmo na ausência de perda de peso ou aumento da massa livre de gordura. Ao longo do tempo, tais adaptações agudas podem alterar significativamente o balanço energético e contribuir para a manutenção do peso corporal saudável e para minimizar a deposição de gordura.

Assim sendo, programas de treinamento com exercícios designados para promover aumento da força muscular são adequados para indivíduos idosos que desejam perder peso.

\section{CONCLUSÃO}

A prescrição de exercícios para se obter o controle de peso deve ser baseada em evidências científicas.

Para idosos com peso dentro da normalidade, o objetivo é a manutenção do peso, a prevenção do acúmulo de gordura corporal e da perda de massa livre de gordura, incluindo o músculo e o osso. Para tanto, é recomendada a realização de atividade aeróbica pelo menos 3 vezes por semana e de atividade com pesos, de 2 a 3 vezes por semana.

Já para idosos obesos, somente o exercício aeróbico não resulta em perdas consideráveis do peso, devendo ser combinado com modificações nos hábitos alimentares.

Há poucas evidências sobre a intensidade ideal dos exercícios para perda de peso, porém, sabe-se que a maioria dos obesos idosos sedentários não consegue sustentar a realização de exercícios aeróbicos de alta intensidade, que promovem, inclusive, riscos cardiovasculares. Portanto, é recomendada para essa população a utilização de exercícios cuja intensidade seja leve ou moderada.

Os benefícios do exercício com peso foram observados somente em treinamentos progressivos de alta intensidade. Se a intenção do treinamento é a modificação da composição corporal mediante a preservação ou o aumento da massa livre de gordura, assim como o aumento da taxa metabólica basal, é recomendado um treinamento de alta intensidade de 2 a 3 vezes por semana.

As evidências epidemiológicas sugerem que a manutenção de uma atividade física regular, ao longo da vida, contribui: para a manutenção ideal do peso, para menores valores de gordura, para maiores valores de densidade óssea e, talvez, para a minimização das perdas de massa muscular acompanhadas pelo processo de envelhecimento.

Os estudos demonstraram que a aplicação de doses adequadas de treinamento de endurance ou de resistência é capaz de reduzir o peso, a gordura e a obesidade central, além de aumentar a massa muscular e a densidade mineral óssea em idosos. Entretanto, o grau dessas mudanças depende de características biológicas individuais, do tipo e da duração do exercício.

O treinamento com pesos pode promover a melhor relação custo/benefício para idosos obesos pela praticidade, pela segurança e pela indução específica de hipertrofia muscular. 
Tabela I: Classificação de sobrepeso e obesidade segundo o Índice de Massa Corporal (IMC).

\begin{tabular}{|l|c|c|}
\hline Classificação & IMC $\left(\mathrm{kg} / \mathrm{m}^{2}\right)$ & Classificação de Obesidade \\
\hline Obesidade Mórbida & $>40,0$ & III \\
\hline Obesidade & $39,9-35,0$ & II \\
\hline Sobrepeso & $34,9-30,0$ & I \\
\hline Normal & $29,9-25,0$ & \\
\hline Abaixo do peso & $24,9-18,5$ & \\
\hline
\end{tabular}

(Adaptado de Executive summary of the clinical guidelines on the identification, evaluation, and treatment of overweight and obesity in adults, 1998).

Tabela II: Limites desejáveis do índice de massa corporal segundo a idade.

\begin{tabular}{|c|c|c|}
\hline \multirow{2}{*}{ Grupo Etário (anos) } & \multicolumn{2}{|c|}{ Índice de Massa Corporal $\left(\mathrm{kg} / \mathrm{m}^{2}\right)$} \\
\cline { 2 - 3 } & Mulheres & $19-24$ \\
\hline $19-24$ & $19-24$ & $20-25$ \\
\hline $25-34$ & $20-25$ & $20-25$ \\
\hline $35-44$ & $21-26$ & $20-25$ \\
\hline $45-54$ & $22-27$ & $20-25$ \\
\hline $55-64$ & $23-28$ & $20-25$ \\
\hline$>65$ & $24-29$ & 25 \\
\hline
\end{tabular}

Fonte: BRAY (1987)

Tabela III: Classificação do risco do sobrepeso baseado na presença dos fatores complicadores: idade, sexo e distribuição anatômica da gordura corporal.

\begin{tabular}{|l|l|}
\hline IMC < 25 & \multicolumn{2}{|l|}{$\begin{array}{l}\text { Com fatores complicadores } \\
\text { Risco Baixo }\end{array}$} \\
\hline $25<$ IMC $<30$ & Com fatores complicadores \\
\hline $\begin{array}{l}\text { Sem fatores complicadores } \\
\text { Risco Baixo }\end{array}$ & Risco Moderado \\
\hline $30<$ IMC < 35 & Com fatores complicadores \\
\hline $\begin{array}{l}\text { Sem fatores complicadores } \\
\text { Risco Moderado }\end{array}$ & Risco Alto \\
\hline $35<$ IMC < 40 & Com fatores complicadores \\
\hline $\begin{array}{l}\text { Sem fatores complicadores } \\
\text { Risco Alto }\end{array}$ & Risco Muito Alto \\
\hline IMC $>40$ & Com fatores complicadores \\
\hline $\begin{array}{l}\text { Sem fatores complicadores } \\
\text { Risco Muito Alto }\end{array}$ & \\
\hline
\end{tabular}

(adaptado de Bray, 1992) 


\section{BIBLIOGRAFIA}

1. AMERICAN COLLEGE OF SPORTS MEDICINE. Position statement on proper and improper weight loss programs MSSE 1998.

2. BALLOR, D.L.; KEESEY, R.E. A meta-analysis of the factors affecting exercise-induced changes in body mass, fat mass and fat-free mass in males and females. Int J Obes 15: 717-726, 1991.

3. BERGMAN, E.A.; BOYUNGS, J.C. Indoor walking program increases lean body composition in older women. J Americ Diet Assoc 91(11): 1433-1435, 1991.

4. BRAY, G.A. Overweight is risking fate. Definition, classification, prevalence and risks. Ann N Y Acad Sci 249: 14-28, 1997.

5. BUTTERWORTH, D.E.; NIEMAN, D.C.; PERKINS, R.; et al. Exercise training and nutrient intake in elderly women. J Am Dietetic Assoc 93: 653-657, 1993.

6. CAMPBELL, W.W.; CRIM, M.C.; YOUNG, V.R.; EVANS, W. J. Am J of Clin Nut 60: 167-175, 1994.

7. DUPLER, T.L.; CORTES, C. Effects of a whole-body resistive training regimen in the elderly. Gerontol 39: 314-319, 1993.

8. EVANS, W.J. Exercise guidelines for the elderly. MSSE 31(1): 12-17, 1999.

9. Executive summary of the clinical guidelines on the identification, evaluation, and treatment of overweight and obesity in adults. J Am Dietetic Assoc 98(10): 11781191, 1998.

10. FIATARONE-SINGH M.A. Body Composition and weight control in older adults. In: LAMB, D.R.; MURRAY, R. (eds). Perspective in Exercise Science and sports medicine: exercise, nutrition and weight control. Carmel: Cooper; p. 378-393, 1998b.

11. Fundação IBGE [Censo demográfico on line]. Rio de Janeiro; 1998. Disponível em http://www.ibge.gov.br [2001 Mar 30].

12. GORAN, M.I.; POEHLMAN, E.T. Total Energy Expenditure and Energy Requirements in healthy elderly persons. Metabol 41(7): 744-753, 1999.

13. GUEDES, D.P.; GUEDES, E.R.P. Controle do peso corporal: composição corporal, atividade física e nutrição. Londrina: Midiograf, 1998.

14. HUNTER, G.R.; WETZSTEIN, C.J.; FIELDS, D.A. et al. Resistance training increases total energy expenditure and free-living physical activity in older adults. J Apply Physiol 89: 977-984, 2000.

15. HURLEY, B.F.; HAGBERG, J.M. Optimizing health in older persons: aerobic or strenght training. Exercise and Sports Sciences Reviews 26: 61-89, 1998.

16. KEIM, N.L.; BARBIERI, T.F.; BELKO, A.Z. The effect of exercise on energy intake and body composition in overweight women. Int J Obes 14: 335-346, 1990.

17. LUDO, M.L.; VAN ETTEN; KLAAS, L.; WESTERTERP, F.R. J.; et al. J Apply Physiol 82(1): 298-304, 1997.

18. MATSUDO, S.M.M. Teorias do Envelhecimento. In: MATSUDO, S.M.M. (eds). Envelhecimento \& atividade física Midiograf, p. 18-22, 2001.

19. MATSUDO, S.; MATSUDO, V. Prescrição e benefícios da atividade física na terceira idade. Rev Bras Cienc Mov 6: 19-30, 1992
20. Mc ARDLE, W.; et al. Fisiologia do Exercício: Energia, Nutrição e Desempenho humano, 4a ed., Guanabara, Koogan. E. U. A., 1998

21. MICHAEL, J.; TOTH, T. BECKETT; ERIC, T. POEHLMAN. Physical activity and the progressive change in body composition with aging: current evidence and research issues. MSSE 31(11), 1999, Suppl:S590-S596.

22. POLLOCK, M.L.; EVANS, W.J. Resistance traaining for health and disease: introduction. MSSE 31(1): 10-11, 1999.

23. NICHOLS, J.F.; OMIZO, D.K.; KATRINA, K.P.; NELSON, K. P. JAGS 41: 205-210, 1993.

24. NIEMAN, D.C.; ONASCH, L.M.; LEE, J.W. The effects of moderate exercise training on nutriente intake in mildly obese women. J Am Dietetic Assoc 90: 1557-1562, 1990.

25. POLLOCK, M.L.; EVANS, W.J. Resistance training for health and disease: introduction. MSSE 1998.

26. PRATLEY, R.; NICKLAS, B.; RUBIN, M.; et al. J Apply Physiol 76(1): 133-137, 1994.

27. RASO, V. Exercícios com pesos para pessoas idosas: a experiência do Celafiscs. Rev Bras Ciên Mov 8(2): 41-49, 2000

28. READY, A.E.; NAIMARK, B.; DUCAS, J.; et al. Influence of walking volume on health benefits in women postmenopause. MSSE 28(9): 1097-1105, 1996.

29. RYAN, A.S.; PRATLEY, R.E.; ELAHI, D.; GOLDBERG, A.P. Resisitive training increases fat-free mass and maintains RMR despitee weight loss in postmenopausal women. J Apply Physiol 79(3): 818-823, 1995.

30. SCHOUERI JUNIOR, R.; RAMOS, L.R.; PAPALÉO NETTO, M. Crescimento populacional: aspectos demográficos e sociais. In: CARVALHO FILHO, E.T.; PAPALÉO NETTO, M. (eds). Geriatria: fundamentos, clínica e terapêutica. Atheneu, p.9-29, 2000.

31. SERDULA, M.K.; MOKDADE, A.H.; WILLIAMSON, D.F. et al. Prevalence of attempting weight loss and strategies for controlling weight. JAMA 1999; 282 (14):1353-1358.

32. SIPILA, S.; SUOMINEN, H. Effects of strength and endurance training on thigh and leg muscle mass and composition in elderly women. J Apply Physiol 78(1): 334-340, 1995.

33. SMITH, D.A.; WITHERS, R.T.; BRINKMAN, M. Resting metabolic rate, body composition and aerobic fitness comparisions between active and sedentary 54-71 year old males. Eur J Clin Nut 53: 434-440, 1999.

34. STEFANICK, M.L.; MACKEY, S.; SHEEHAN, M. et al. N Eng J Med 339: 12-20, 1998.

35. TOTH, M.J.; BECKETT, T.; POEHLMAN, E.T. Physical activity and the progressive change in body composition with aging: current evidence and research issue. MSSE 31(11), 1999, Suppl: S590 - S596.

36. VERAS, R.P.; et al. Crescimento da população idosa no Brasil: transformações e conseqüências na sociedade. Rev Saúde Pub 21: 225-233, 1987.

37. YOUNG, D.R.; APPEL, L.J.; JEE, S.; et al. The effects of aerobic exercise and T'ai Chi on blood pressure in older people: results of a randomized trial. JAGS 42: 277-284, 1999. 